\title{
Identification of Fusarium oxysporum Fungus in Wheat Based on Chemical Markers and Qualitative GC-MS Test
}

\author{
Carlos E. R. Senes, ${ }^{a}$ Nayara C. Saldan, ${ }^{a}$ Willian F. Costa, ${ }^{a}$ Terezinha I. E. Svidzinski ${ }^{b}$ \\ and Cláudio C. Oliveira*,a \\ ${ }^{a}$ Departamento de Química, Centro de Ciências Exatas, Universidade Estadual de Maringá, \\ Avenida Colombo, 5790, Zona 7, 87020-900 Maringá-PR, Brazil \\ ${ }^{b}$ Departamento de Análises Clínicas e Biomedicina, Centro de Ciências da Saúde, \\ Universidade Estadual de Maringá, 87020-900 Maringá-PR, Brazil
}

\begin{abstract}
Fusarium oxysporum is usually found in foods such as corn, wheat, soybeans and barley, and can cause economic losses and risks to health in humans and animals due to the production of mycotoxins. The conventional microbiological test for fungal identification is based on morphological characteristics, requires specialists, and it is very time-consuming as it is necessary to carry out a culture of the fungus for two weeks before microorganism identification. Nowadays, researchers have made efforts to develop alternative analytical methods with advantages such as shorter time of analysis and better accuracy and reproducibility when compared to microbiological methods. In this way, in the present work, a qualitative analytical method to detect the secondary metabolites of Fusarium oxysporum in culture media by gas chromatography coupled to mass spectrometry (GC-MS) was developed with the intention of relating the presence of these chemical compounds to contamination by the fungus. The method comprised liquid-liquid or liquid-solid extractions with ethyl acetate from liquid and solid culture media, respectively, followed by GC-MS analysis, which was able to identify the presence of fusaric acid, 13-socosenamide, 5-(4-hydroxybutyl)-2-pyridinecarboxylic acid and toxin HT-2. The presence of these compounds was confirmed by high-performance liquid chromatography coupled to mass spectrometry quadrupole time of flight (HPLC-MS/QTOF).
\end{abstract}

Keywords: chemical markers, Fusarium oxysporum, fungus, gas chromatography

\section{Introduction}

Fungi are microorganisms that present chromosomes and nucleoli enclosed in a cell membrane, being classified as eukaryotic. They can be unicellular or multicellular, and are classified as heterotrophic. Reproduction usually occurs through spores and can be sexual or asexual. ${ }^{1}$ Organic matter and humidity are important for their growth, but they can survive in dry environments through the production of aridity-resistant spores. ${ }^{1}$

Toxigenic fungi produce toxic secondary metabolites (mycotoxins) during their life, which can be carcinogenic in human and animals. ${ }^{2}$ Fungi can enter the food chain through food contamination, which occurs by the contact with spores present in the environment, particularly in the soil, or during harvesting, storage, and transport, ${ }^{3}$ and

*e-mail: ccoliveira@uem.br consecutively contaminate animals and the humans that consume them. ${ }^{4}$

The fungi belonging to the genus Fusarium are filamentous, and are able to produce different types of mycotoxin, and when in contact with the cereals, can cause health problems. Among the main mycotoxins produced by this genus are the fumonisins, trichothecenes (vomitoxin, T-2, among others), zearalenone, moniliformin, and fusaric acid. ${ }^{5}$ The species Fusarium oxysporum is responsible for at least 20 different diseases, occurring in more than 120 species of plants. It invades the water conductive vessels of the plant and promotes vascular wilting, leading to premature death of the plant. Corn, wheat, soy, and barley are among the main plants attacked by this fungus. In humans, Fusarium is known as an etiologic agent of opportunistic infections, such as onychomycosis, locally invasive infections or in the case of immunocompromised individuals, disseminated 
infections, including mycotoxicosis and allergic diseases (sinusitis), ${ }^{6}$ making the control of fungi in foods a necessity; furthermore, food contamination leads to economic losses, which justify the development of fungus identification methods in food. ${ }^{5}$

Conventional microbiological methods used for the identification of $F$. oxysporum require a long time for analysis, mainly due to the need to cultivate the fungus, usually for 15 days in culture media for subsequent macroscopic and microscopic analysis, which requires specialized people with practice in the identification of fungal agents. ${ }^{?}$

Thus, the possibility of identifying the fungus chemically through the development of analytical methods that can relate the presence of certain substances and chemical markers to fungus contamination becomes attractive, mainly because there is no need to cultivate the fungus, leading to a shorter analysis time, no need to rely on highly specialized people in the identification of macroscopic and microscopic characteristics of fungi, and having greater sensitivity and accuracy. ${ }^{8,9}$

Chromatography coupled to mass spectrometry has been explored to separate and identify fungal metabolites ${ }^{9,10}$ due to the sensitivity, precision and accuracy, and the possibility to identify chemical substances unequivocally in complex matrices. ${ }^{11}$ In this way, the objective of the present work is to develop a qualitative methodology using gas chromatography coupled to mass spectrometry (GC-MS) to separate and identify chemical substances produced by $F$. oxysporum in traditional culture media and in wheat samples that can be used as chemical markers of the fungus, opening the possibility to develop an analytical method to identify the fungus chemically with greater speed and sensitivity.

\section{Experimental}

\section{Preparation of culture media}

Potato dextrose agar (PDA)

Approximately $42.0 \mathrm{~g}$ of PDA medium (HIMEDIA, Mumbai, India) was weighed and transferred to an Erlenmeyer flask containing $1 \mathrm{~L}$ of deionized water. After gentle stirring, the mixture was heated in a microwave oven until complete solubilization and autoclaved for later use.

\section{Czapek-Dox agar (CDA)}

Approximately $49.0 \mathrm{~g}$ of CDA medium (HIMEDIA, Mumbai, India) was weighed and transferred to an Erlenmeyer flask containing $1 \mathrm{~L}$ of deionized water. After gentle stirring, the mixture was heated in a microwave oven until complete solubilization, the $\mathrm{pH}$ was adjusted to 5.5, and it was autoclaved for later use.

\section{Fungal culture conditions in PDA and Czapek-Dox media}

$50.0 \mathrm{~mL}$ of PDA culture medium was prepared and autoclaved. Approximately $7.0 \mathrm{~mL}$ was transferred to test tubes. Then, strains of $F$. oxysporum, supplied by the Laboratory of Medical Mycology of UEM (Universidade Estadual de Maringá, Brazil), were seeded in the test tubes. After 15 days of incubation at $25^{\circ} \mathrm{C}$, a new seeding was performed on three disposable Petri dishes $(150 \times 15 \mathrm{~mm})$ with the same culture medium. Another three Petri dishes were kept without seeding as controls (blank). They were also incubated at $25^{\circ} \mathrm{C}$ for 15 days. ${ }^{12}$

$50.0 \mathrm{~mL}$ of PDA culture medium was prepared in an Erlenmeyer flask, and autoclaved after complete microwave-assisted solubilization. Then, it was poured into six disposable Petri dishes $(150 \times 15 \mathrm{~mm})$, which had previously been autoclaved..$^{12}$ After complete solidification, a new seeding of the $F$. oxysporum fungus was performed using the test tubes, which were incubated for 15 days. Only three Petri dishes were re-seeded; the other three were used as controls. The plates were incubated at $25^{\circ} \mathrm{C}$ for 15 days. ${ }^{8-12}$

After 15 days of incubation, six disks of the culture of approximately $5 \mathrm{~mm}$ diameter were placed in two Erlenmeyer flasks containing $200 \mathrm{~mL}$ of Czapek-Dox liquid medium. The procedure was performed behind a Bunsen burner flame to avoid contamination. Cutouts were performed using pipettor and blade tips. Two control solutions (blanks) were also prepared under the same conditions. ${ }^{8-12}$ The Erlenmeyer flasks were kept in an incubator at $25^{\circ} \mathrm{C}$ with orbital shaking at $70 \mathrm{rpm}$ for 15 days. Afterwards, the cultures were filtered with glass funnel filter paper to remove the mycelia. The filtrate was analyzed under a microscope to confirm the absence of contamination in the culture, and then filtered through a membrane with a porosity of $0.45 \mu \mathrm{m}$ and $47 \mathrm{~mm}$ in diameter (sterile extract, ESE) and subjected to extraction. The same procedure was performed for the control. ${ }^{8-12}$

Extraction of the secondary metabolites from solid and liquid media

\section{Solid medium}

Agars on Petri dishes were cut into small cubes $\left(0.3 \mathrm{~cm}^{3}\right)$ using a scalpel blade. The contents of each plate were transferred to a falcon tube and frozen at $-80{ }^{\circ} \mathrm{C}$ overnight, in order to weaken the fungus cell wall, which enhanced solvent penetration into the cell and, therefore, 
the extraction efficiency. Afterward, the material was transferred to an amber glass vial containing $15.0 \mathrm{~mL}$ of ethyl acetate (Panreac, Barcelona, Spain). Vials were sonicated for $30 \mathrm{~min}$, facilitating the extraction process, and left in an orbital shaker at $100 \mathrm{rpm}$ for $30 \mathrm{~min},{ }^{7,13}$ in order to further enhance the extraction. The sonication procedure was repeated, and the solution was filtered through a paper filter attached to a glass funnel, then through a Millipore membrane with $0.45 \mu \mathrm{m}$ pore size and $47 \mathrm{~mm}$ diameter (Bedford, MA, USA). The extracts were stored under refrigeration in amber bottles until GC-MS analysis.

\section{Liquid medium}

$40.0 \mathrm{~mL}$ of ESE and $15.0 \mathrm{~mL}$ of ethyl acetate were added to a separating funnel, which was shaken vigorously, and the system was allowed to stand for a few minutes until phase separation occurred. The organic phase was collected, and the liquid-liquid extraction in the aqueous phase was repeated three more times, always with $15.0 \mathrm{~mL}$ of ethyl acetate. The four organic portions $(60.0 \mathrm{~mL})$ were combined and left in an amber flask until GC-MS analysis. ${ }^{14,15}$

Application of the analytical methods for fungal chemical marker identification in food samples

Assays were performed with samples contaminated with $F$. oxysporum inoculum, control samples (without contamination) and samples that had been maintained in poor storage conditions in order to achieve natural contamination with $F$. oxysporum. Wheat, which is a natural substrate for fungi growth, ${ }^{16}$ was purchased in local markets of the city of Maringá (Paraná, Brazil) and analyzed in triplicate.

The suspension for the preparation of the inoculum was obtained from a culture of $F$. oxysporum in PDA, with the aid of a sterile $0.85 \%$ saline solution prepared by adding $8.5 \mathrm{~g}$ of sodium chloride (Panreac, Barcelona, Spain) to $1 \mathrm{~L}$ of deionized water, which was used for handling samples and diluted microorganisms. The suitable concentration of microorganism suspension was determined in accordance with the literature ${ }^{17}\left(1 \times 10^{7}\right.$ colony forming units $\left.(\mathrm{CFUs})\right)$. The estimated number of CFUs in a suspension of conidia was obtained by microscopy counting in a Neubauer chamber. ${ }^{16}$

For samples inoculated with the fungus, approximately $10.00 \pm 0.01 \mathrm{~g}$ of feed samples (both in natural and milled forms (particle size between 1.70 and $1.20 \mathrm{~mm}$ )) were weighed, poured into glass tubes with caps and sterilized in an autoclave. Next, samples were transferred to disposable Petri dishes $(90 \times 15 \mathrm{~mm})$ in a sterile environment.
Approximately $1.0 \mathrm{~mL}$ of inoculum $\left(1 \times 10^{7} \mathrm{CFUs}\right)$ was added to each plate, which was incubated at $25{ }^{\circ} \mathrm{C}$ in a suitable incubator for 60 days. ${ }^{16}$ For control and naturally contaminated samples, the same procedures as those used for inoculated samples were performed, but without the addition of fungal inoculum and with the addition of saline solution for poorly stored samples, which were placed in an external environment and subjected to weather conditions instead of an incubator.

After 60 days of incubation, all samples were transferred to falcon tubes, frozen at $-80{ }^{\circ} \mathrm{C}$ overnight in order to weaken the cell walls, and transferred to amber glass vials containing $15.0 \mathrm{~mL}$ of ethyl acetate, in order to equalize the matrix conditions for the extraction step, which was the same process carried out for fungus cultivation on PDA medium..$^{14-16}$ For extraction, vials were sonicated for $30 \mathrm{~min}$ and left in an orbital shaker at $100 \mathrm{rpm}$ for $30 \mathrm{~min}$. The sonication procedure was repeated once more and the solutions were filtered through a Millipore membrane with $0.45 \mu \mathrm{m}$ pore size and $47 \mathrm{~mm}$ diameter (Bedford, MA, USA) ${ }^{14,15}$ and analyzed by GC-MS.

\section{GC-MS analysis}

The identification of secondary metabolites produced by $F$. oxysporum was achieved using a gas chromatograph (Focus GC, Thermo-Finnigan, USA) coupled to a mass spectrometer (DSQ II-Thermo-Finnigan, USA) equipped with DB-5 (5\% phenyl-95\% methylpolysiloxane) capillary column of fused silica $(30 \mathrm{~m} \times 0.25 \mathrm{~mm}$ i.d. $\times 0.25 \mu \mathrm{m}$, J\&W Scientific, USA). The mass spectrometer was equipped with an electron ionization source, quadrupole mass analyzer and a data acquisition system using Xcalibur ${ }^{\circledR}$ software, which included the NIST MS Search Program version 2.0 spectrum library.

For separation, temperature programming was set at $40{ }^{\circ} \mathrm{C}(2 \mathrm{~min})$ and the ramp at $3{ }^{\circ} \mathrm{C} \mathrm{min}^{-1}$ to $300^{\circ} \mathrm{C}(5 \mathrm{~min})$; other chromatographic conditions can be seen in Table $1 .{ }^{18}$

Table 1. Chromatographic conditions for secondary metabolites analysis by GC-MS

\begin{tabular}{lc}
\hline \multicolumn{2}{c}{ Chromatographic conditions } \\
\hline Injector temperature $/{ }^{\circ} \mathrm{C}$ & 270 \\
Injection mode & splitless \\
Gas & helium \\
Flow rate $/\left(\mathrm{mL} \mathrm{min}^{-1}\right)$ & 1.0 \\
Injected volume / $\mu \mathrm{L}$ & 1.0 \\
Voltage / eV & 70 \\
Scan range $\mathrm{m} / \mathrm{z}$ & $43-450$ \\
\hline
\end{tabular}


High-performance liquid chromatography-MS quadrupole time of flight (HPLC-MS/QTOF) analysis

The confirmation of secondary metabolites produced by $F$. oxysporum and identified by the GC-MS was achieved using Shimadzu maXis Impact (Kyoto, Japan) HPLC equipment coupled to a quadrupole timeof-flight (micrOTOF-Q ${ }^{\mathrm{TM}}$, Bruker Daltonik GmbH, Bremen, Germany) orthogonal accelerated QTOF mass spectrometer, equipped with an electrospray ionization source (ESI). Separation of analytes was performed using a XRODSIII $(150 \mathrm{~mm} \times 2 \mathrm{~mm} \times 2.2 \mu \mathrm{m})$ column from Shimadzu (Kyoto, Japan). Acidified deionized water (A) $(0.1 \%$ formic acid $\mathrm{v} / \mathrm{v})$ and acidified acetonitrile (B) $(0.1 \%$ formic acid v/v) (Merck Millipore, Darmstadt, Germany) were used as mobile phases A and B, respectively. The gradient was executed as follows: 5\% solvent B 0-2 min; increased to $90 \%$ B 2-15 min; 90\% B until $20 \mathrm{~min}$; 5\% B 20-21 min and 5\% B until 25 min. Parameters for analysis were set using positive ion mode (ESI+), with spectra acquired over a mass range of $m / z 70-1300 \mathrm{Da}$. The optimized parameters for ESI-MS were: capillary voltage, $4.5 \mathrm{kV}$; drying gas temperature, $190^{\circ} \mathrm{C}$; drying gas flow, $10.0 \mathrm{~L} \mathrm{~min}^{-1}$, and nebulizing gas pressure, $4 \times 10^{5} \mathrm{~Pa}$. Besides that, additional automatic MS/MS experiments were performed $(\mathrm{m} / \mathrm{z}, 0-1300)$ with nitrogen $\left(\mathrm{N}_{2}\right)$ as the collision gas and adjusting the collision energy values as follows: $\mathrm{m} / \mathrm{z}$ 0-100, $20 \mathrm{eV} ; \mathrm{m} / \mathrm{z} 101-500,30 \mathrm{eV} ; \mathrm{m} / \mathrm{z}$ 501-1000, $35 \mathrm{eV}$. The MS data were processed through Data Analysis 4.0 software (Bruker Daltonics, Bremen, Germany), which provided a list of possible elemental formulas by using the Generate Molecular Formula ${ }^{\mathrm{TM}}$ editor. The widely accepted accuracy for confirmation of elemental compositions has been established as 5 ppm. ${ }^{9}$

\section{Results and Discussion}

\section{Morphological analyses}

The macroscopic morphological analyses of the F. oxysporum cultivated in PDA medium confirmed the presence of the fungus without contamination as it was verified abundant cottony mycelia with colony color and pigmentations from pink to purple, which are structures characteristic of the fungus. In the microscopic morphological analysis, it was possible to verify the presence of septate, fusiform and curved macroconidia, confirming the identification of the fungus and showing that there was no contamination by other microorganisms (Figure 1).

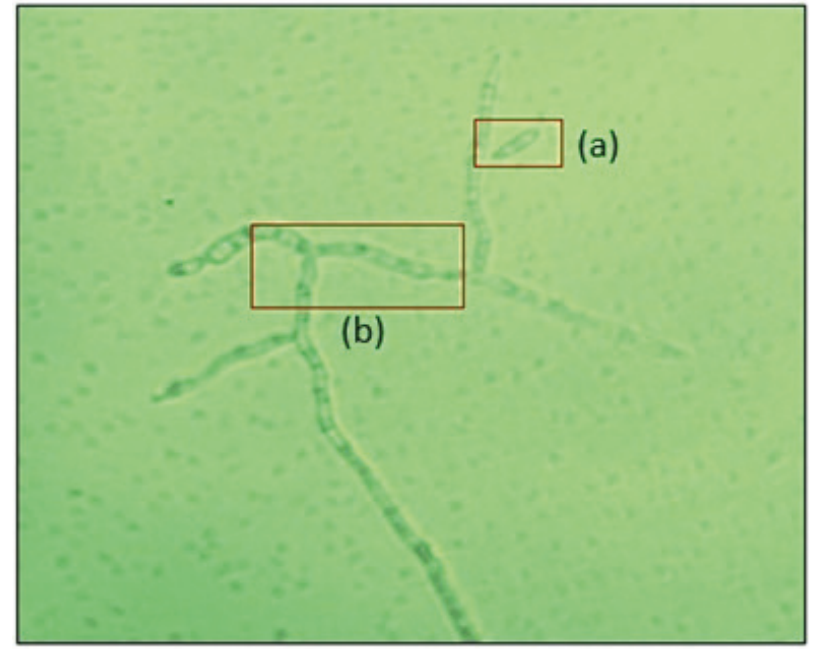

Figure 1. Micrograph of a Fusarium oxysporum. (a) Septum macroconidia; (b) septate hyaline hyphae.

Identification of the metabolites of the fungus cultivated in liquid culture medium by GC-MS

Stability of the GC-MS signals was verified under the selected operating conditions according to Table 1 , and it was considered as signal only peaks with S/R higher than 3.3 according to the literature recommendations; ; 19 the detection limits of the equipment were 0.37-19.19 and 1.24-63.33 $\mu \mathrm{g} \mathrm{kg}^{-1}$, but the values can suffer fluctuations depending on the sample and metabolite. ${ }^{19}$ During the GC-MS analysis of the ethyl acetate extracts from liquid medium it was possible to observe analytical signals at retention times of 33.99, 34.96, 36.33 and $45.15 \mathrm{~min}$ (Figure 2).

Mass spectra of these peaks (Figure 3) allowed to do the peaks attribution to 13 -docosenamide $(\mathrm{m} / \mathrm{z} 337)$, fusaric acid ( $m / z, 179), 5$-(4-hydroxybutyl)-2-pyridinecarboxylic acid $(\mathrm{m} / \mathrm{z}, 195)$, and cyclopentane 1,1'-[3-(2-cyclopentyl ethylidene)-1,5-pentanediol]-bis ( $\mathrm{m} / \mathrm{z}$ 302), respectively, as the mass spectrum of the peak with retention time of $33.99 \mathrm{~min}$ presented the base peak the ion of $\mathrm{m} / \mathrm{z} 135$ assigned to the $\mathrm{C}_{8} \mathrm{H}_{9} \mathrm{NO}$ fragment, and the ion of $\mathrm{m} / z 149$ indicating the loss of a $\mathrm{CO}$ molecule from the ion of $m / z 177$, according to fragmentation proposals in the literature for the compound. ${ }^{5,8,19-22}$ Furthermore, the loss of $\mathrm{CH}_{2}$ is evident by the difference of $\mathrm{m} / \mathrm{z} 14$ between the ions of $m / z 149$ and 135, which show the highest intensity. The mass spectrum of the peak with retention time of $34.96 \mathrm{~min}$ was attributed to the mycotoxin fusaric acid $(\mathrm{m} / z \mathrm{179})$, as the ion of $m / z, 164$ indicated the loss of methyl fragment and the ion fragment of $\mathrm{m} / \mathrm{z} 136$ indicated the loss of $\mathrm{C}_{2} \mathrm{H}_{4}{ }^{5}{ }^{5,6,21}$ The mass spectrum of the peak with retention time of 36.33 min was attributed to 5-(4-hydroxybutyl)2-pyridinecarboxylic acid $(\mathrm{m} / \mathrm{z}, 195)$, which is a derivative of 


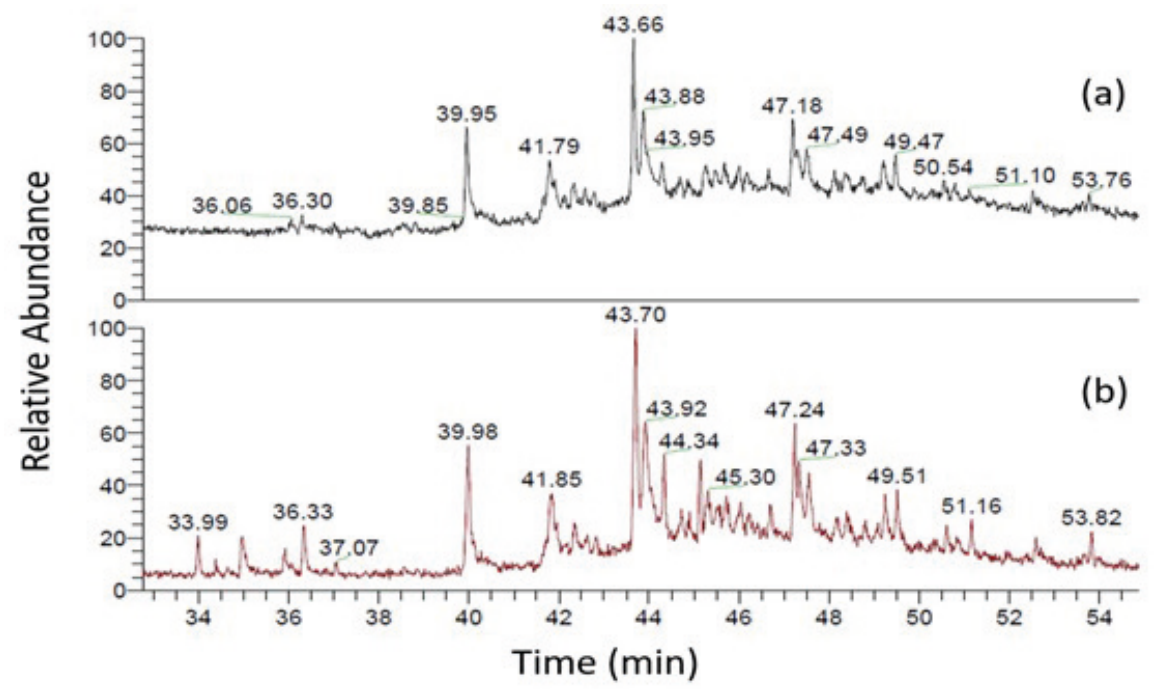

Figure 2. Chromatograms of the extract of the fungus cultivated in liquid medium after extraction with ethyl acetate: (a) control; (b) sample. Injection of $1.0 \mu \mathrm{L}$ of sample in the splitless mode with ionization energy of $70 \mathrm{eV}$.

fusaric acid, and the ions of $\mathrm{m} / \mathrm{z} 164$ and 122 indicated the loss of $\mathrm{CH}_{3} \mathrm{O}$ and $\mathrm{C}_{4} \mathrm{H}_{9} \mathrm{O}$ fragments, respectively. The mass spectrum of the peak eluted at $45.15 \mathrm{~min}$ was attributed to the compound cyclopentane 1,1'-[3-(2-cyclopentyl ethylidene)-1,5-pentanediol]-bis, which is a cyclopentane derivative originating from fatty acids, the primary metabolites. The ions of $\mathrm{m} / \mathrm{z} 109$ and 139 can be attributed to the losses of $\mathrm{C}_{8} \mathrm{H}_{13}$ and $\mathrm{C}_{10} \mathrm{H}_{19}$, respectively. ${ }^{19,20}$ Furthermore, all the compounds attributions were confirmed by the HPLC-MS/QTOF analysis used as the comparative method.

It should be stressed that after extraction in the solid part of the culture it was possible to identify the fatty acids 9-hexadecenoic, 9,12-octadecadienoic, 9-octadecenoic and oleic acids that cannot be considered chemical markers for F. oxysporum.

\section{Identification of the metabolites of the fungus grown in solid medium by GC-MS}

The extract of the fungus cultivated in PDA and its respective blank were submitted to GC-MS analysis under the same instrumental conditions as the fungus extract cultivated in a liquid medium. Comparing the chromatograms of the control and the sample (Figure 4), it was possible to identify signals at the retention times 36.04 , $36.45,36.76,40.13,41.17,43.87,44.09,47.42$, 47.76 and $57.15 \mathrm{~min}$. Through the analysis of the mass spectra of the signals at 40.13, 43.87, 44.09, 47.42, 47.76 and $57.15 \mathrm{~min}$, it was verified that these compounds were not derived from the metabolism of the fungus; they probably originated from the culture medium and from contaminations in the system.

Mass spectra of the compounds eluted at 36.04, 36.45, 36.76 and 41.17 min (Figure 5) allowed identification of the compounds isobutyl (perhydro-5-oxo-2-furyl) propionate, fusaric acid, toxin HT-2 and spiro[4,5]dec7-ene, 1,8-dimethyl-4-(1-methyl phenyl). The signal at $36.04 \mathrm{~min}$ could be attributed to isobutyl (perhydro5-oxo-2-furyl)propionate as the presence of the ions of $\mathrm{m} / \mathrm{z}, 141$ and 85 represent the losses of $\mathrm{C}_{4} \mathrm{H}_{9} \mathrm{O}$ and $\mathrm{C}_{4} \mathrm{H}_{5} \mathrm{O}_{2}$, respectively. The compound eluted at $36.45 \mathrm{~min}$ was assigned to fusaric acid, as the ions of $\mathrm{m} / \mathrm{z}, 164,136$ and 121 indicated the losses of $\mathrm{CH}_{3}, \mathrm{C}_{2} \mathrm{H}_{4}$ and $\mathrm{C}_{4} \mathrm{H}_{10}$ fragments from fusaric acid or $\mathrm{CH}_{3}$ from the ion of $\mathrm{m} / z$, 136. The substance eluted at $36.76 \mathrm{~min}$ was attributed to toxin HT-2. The ions of $m / z, 206,191$ and 57 could be attributed to fragments $\mathrm{C}_{13} \mathrm{H}_{18} \mathrm{O}_{2}, \mathrm{C}_{12} \mathrm{H}_{15} \mathrm{O}_{2}$, and $\mathrm{C}_{4} \mathrm{H}_{9}$, respectively. The compound eluted at $41.17 \mathrm{~min}$ could be attributed to spiro[4,5]dec-7-ene, 1,8-dimethyl-4-(1-methyl phenyl) as the ions of $m / z 119$ and 161 indicate the losses of the fragments $\mathrm{C}_{6} \mathrm{H}_{13}$ and $\mathrm{C}_{3} \mathrm{H}_{7}$, respectively. ${ }^{12}$ In addition, the same fatty acids were found in the solid part of the liquid medium. Again, all the compounds were confirmed by HPLC-MS/QTOF analysis.

Identification of fungus metabolites in wheat samples

In the samples left under poor storage conditions and those with fungus inoculum, it was possible to observe the macroscopic characteristics of the fungus: white mycelium with small rosy variations indicating that the fungus was present in the food. Chromatographic analysis of the extracts of these samples indicated the presence of analytical signals at the retention times of 52.36, 53.23, 58.42, 58.59, 58.84 and 78.89 min (Figure 6).

Peaks eluted at 52.36, 58.42, 58.59, and $58.84 \mathrm{~min}$ were indicated as fatty acids 9-hexadecenoic, 

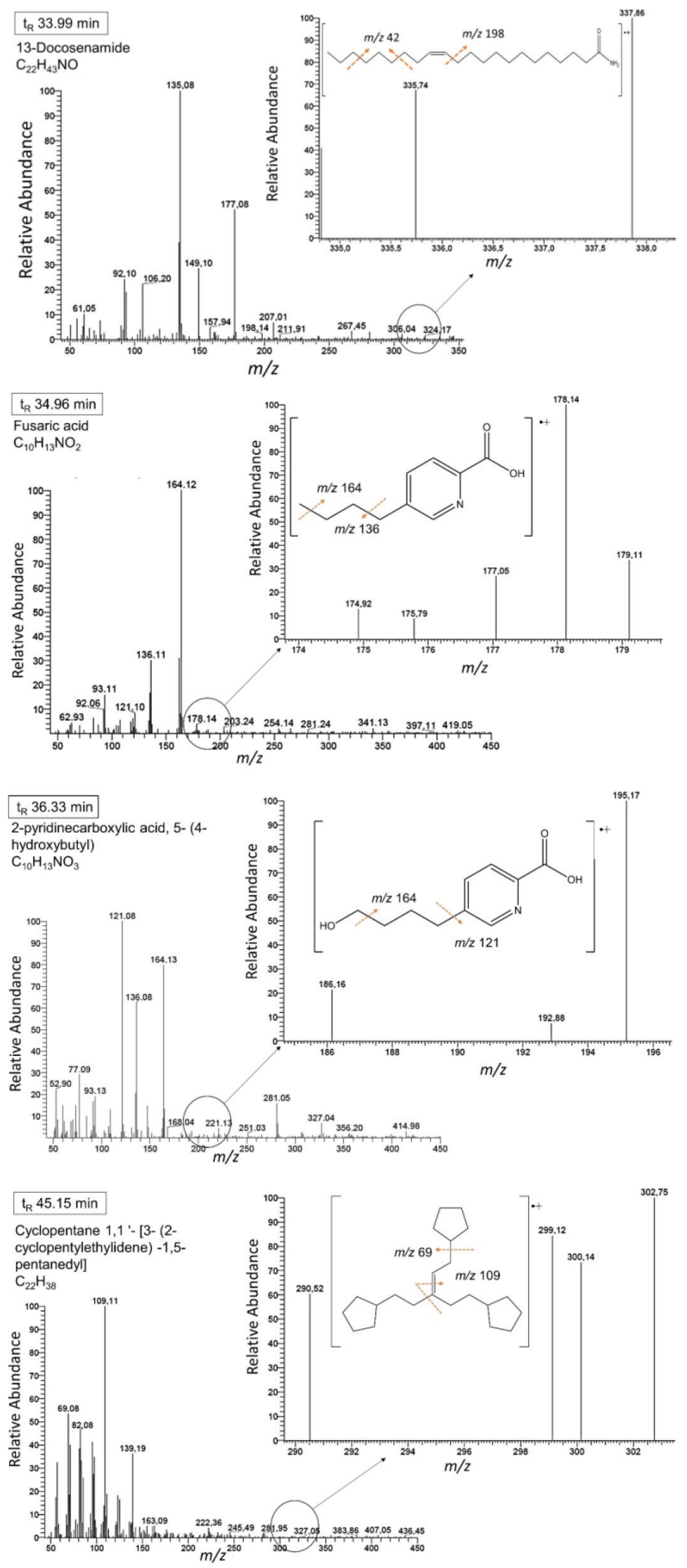

Figure 3. Mass spectra of the peaks eluted at 33.99; 34.96; 36.33 and 45.15 min, after the extraction from liquid medium with ethyl acetate. Injection of $1.0 \mu \mathrm{L}$ of sample in the splitless mode with ionization energy of $70 \mathrm{eV}$ and scanning range between $\mathrm{m} / z$, 43-450. 


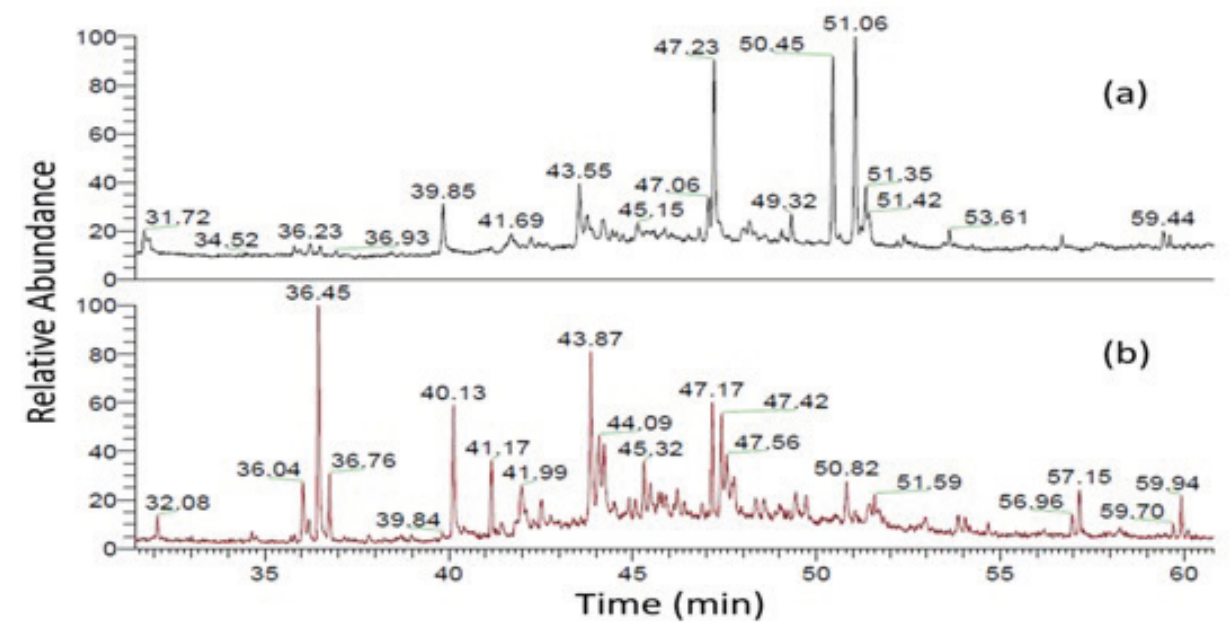

Figure 4. Chromatograms of culture extracts in the solid medium: (a) control; (b) sample. GC-MS in splitless mode with ionization $70 \mathrm{eV}$.

9,12-octadecadienoic, 9-octadecenoic and oleic acids and they are not $F$. oxysporum markers, as they are present in the cultures of other fungi. The peak with the retention time of 53.23 min was attributed to the compound 1,3,5(10)-oestratrien-17 $\beta$-ol, as its mass spectrum (Figure 7) presented the ions of $m / z, 129$ and 72 that could be attributed to the fragments of $\mathrm{C}_{8} \mathrm{H}_{17} \mathrm{O}$ and $\mathrm{C}_{5} \mathrm{H}_{12}$, respectively. Furthermore, the identified compound belongs to the class of estradiols, and is commonly related to zearalenone mycotoxin. ${ }^{14}$

The peak eluted at 78.89 min was identified as dehydroergosterol 3,5-dinitrobenzoate (Figure 7), as the ion fragment of $\mathrm{m} / \mathrm{z} 376$ indicates the loss of a $\mathrm{C}_{12} \mathrm{H}_{22} \mathrm{O}_{3}$. Ergosterol and its derivatives are components of the plasmatic membrane of fungi and are commonly used as indicators of the presence of fungi of the genus Fusarium. ${ }^{16,23}$

The found compounds (Table 2) are known as metabolites of $F$. oxysporum, and they were confirmed by HPLC-MS/QTOF analysis; the presence of at least one of them is sufficient to classify the food sample as inappropriate for human consumption in a rapid screening test as proposed here. The number of secondary metabolites present in a sample depends on several factors such as food composition, time after fungal contamination and storage conditions. Taking this into account, the proposed qualitative analytical method can be used as a rapid screening test for $F$. oxysporum in the tested sample without the necessity for microbial cultures, even without the presence of all the chemical markers. The difference between the chemical compounds found in the standard culture media and in the food samples is associated with the nutrients available in the medium; furthermore, the cultivating time for the samples was 60 days; a longer period may be necessary for the adaptation of the fungus to the medium. It should be stressed that our research group have already verified that fungus chemical markers change as a function of the medium and the incubation time. ${ }^{9}$

Finally, the present work presents the main secondary metabolites produced by $F$. oxysporum cultivated in PDA and Czapek-Dox agar, and in wheat samples, which are extractable by ethyl acetate, an important information that was confirmed by a recognized identification method.

\section{Conclusions}

When ethyl acetate extraction was applied to the liquid fraction of the Czapek-Dox medium, two mycotoxins from $F$. oxysporum were identified (13-docosenamide; 5-(4-hydroxybutyl)-2-pyridinecarboxylic acid) and one derivate from fatty acids (1,1'-[3-(2-cyclopentyl ethylidene)-1,5-pentanediol]-bis). When the same extraction was done in the solid portion, three fatty acids were identified (9-hexadecenoic acid, 9,12-octadecadienoic acid and 9-octadecenoic acid). In the ethyl acetate extract from PDA solid medium, two F. oxysporum mycotoxins (fusaric acid and toxin HT-2) and two compounds that are related in the literature with the fungus (spiro[4,5]dec7-ene, 1,8-dimethyl-4-(1-methyl phenyl) and isobutyl (perhydro-5-oxo-2-furyl)propionate) were identified. In the ethyl acetate extracts from the wheat samples, the presence of two compounds related to the Fusarium genus (1,3,5(10)-oestratrien-17 $\beta$-ol and dehydroergosterol 3,5-dinitrobenzoate) and the same three fatty acids as were found in the solid portion of the Czapek-Dox medium was verified. Thus, it is possible to conclude that the presence of these compounds in the wheat samples is a qualitative indication of the contamination of the food sample by the fungus, indicating the possibility of identifying the fungus in the sample by using a fast chemical analysis by liquid 

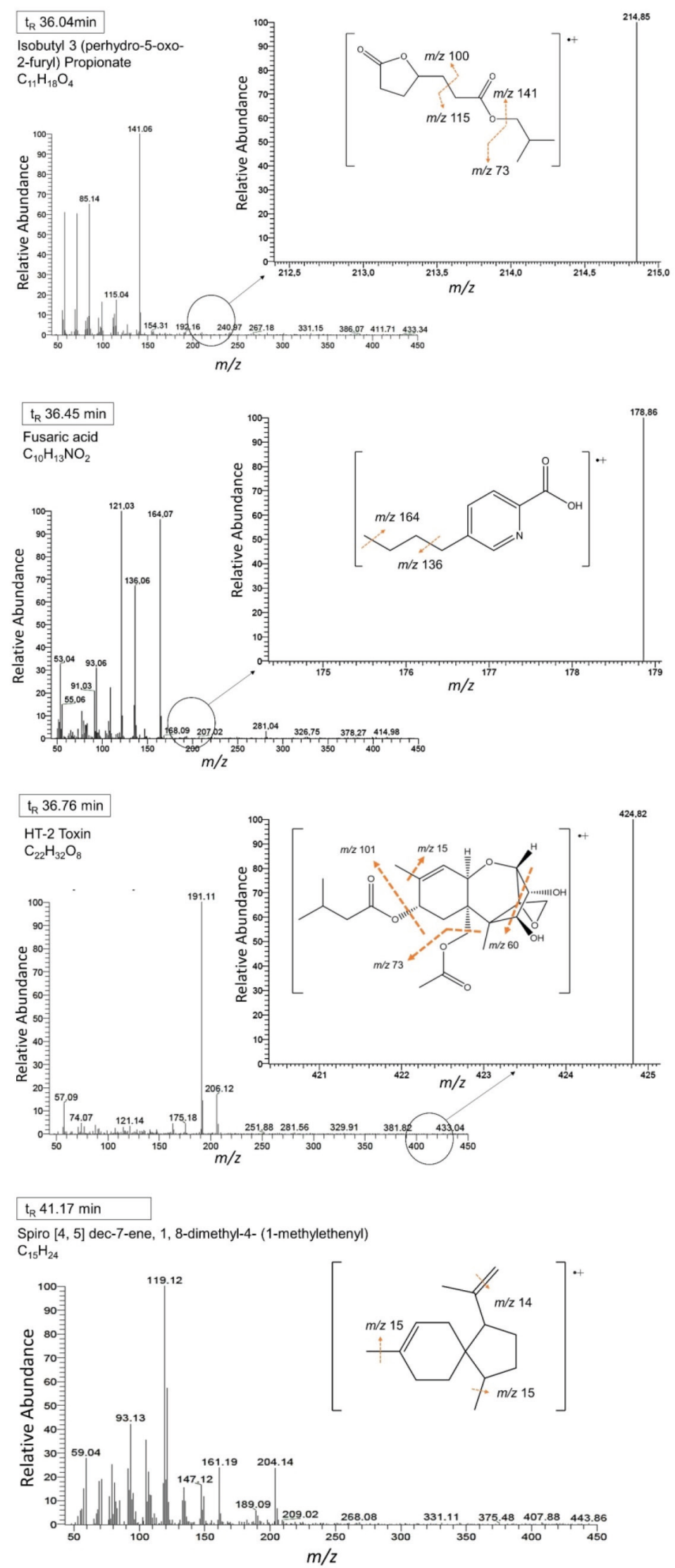

Figure 5. Mass spectrum of peaks with retention times of 36.04, 36.45, 36.76 and $41.17 \mathrm{~min}$. Data obtained after analytes extraction from the solid culture of the fungus and with the GC-MS in the splitless mode with ionization $70 \mathrm{eV}$ and scanning range between $\mathrm{m} / z$ 43-450. 


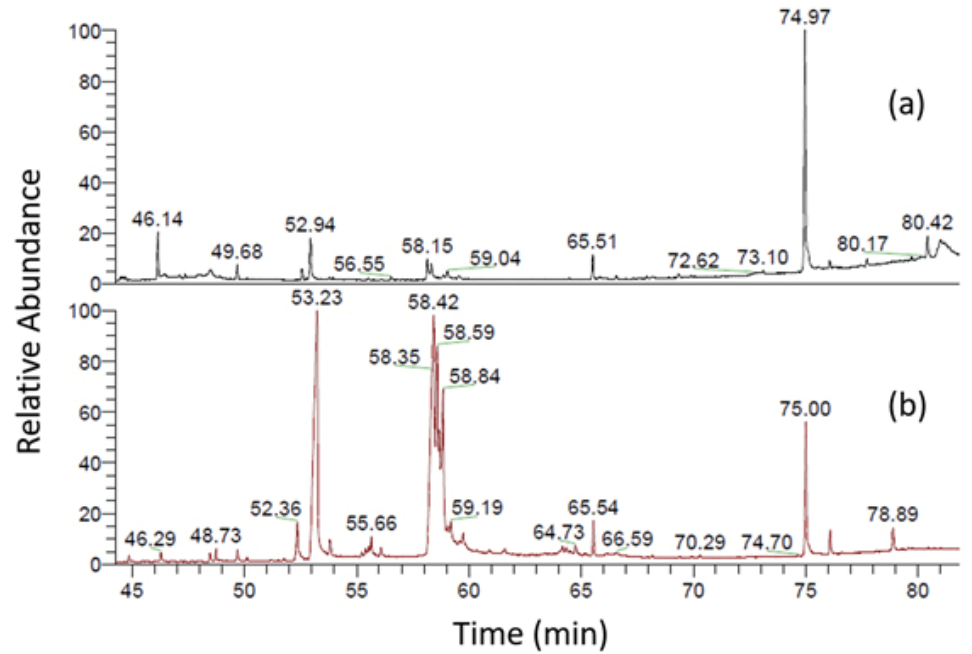

Figure 6. Chromatogram of grain wheat with Fusarium oxysporum inoculums (a) and subjected to bad storage conditions (b) for 60 days. GC-MS in splitless mode with ionization $70 \mathrm{eV}$ and scanning range between $\mathrm{m} / \mathrm{z}, 43-450$.
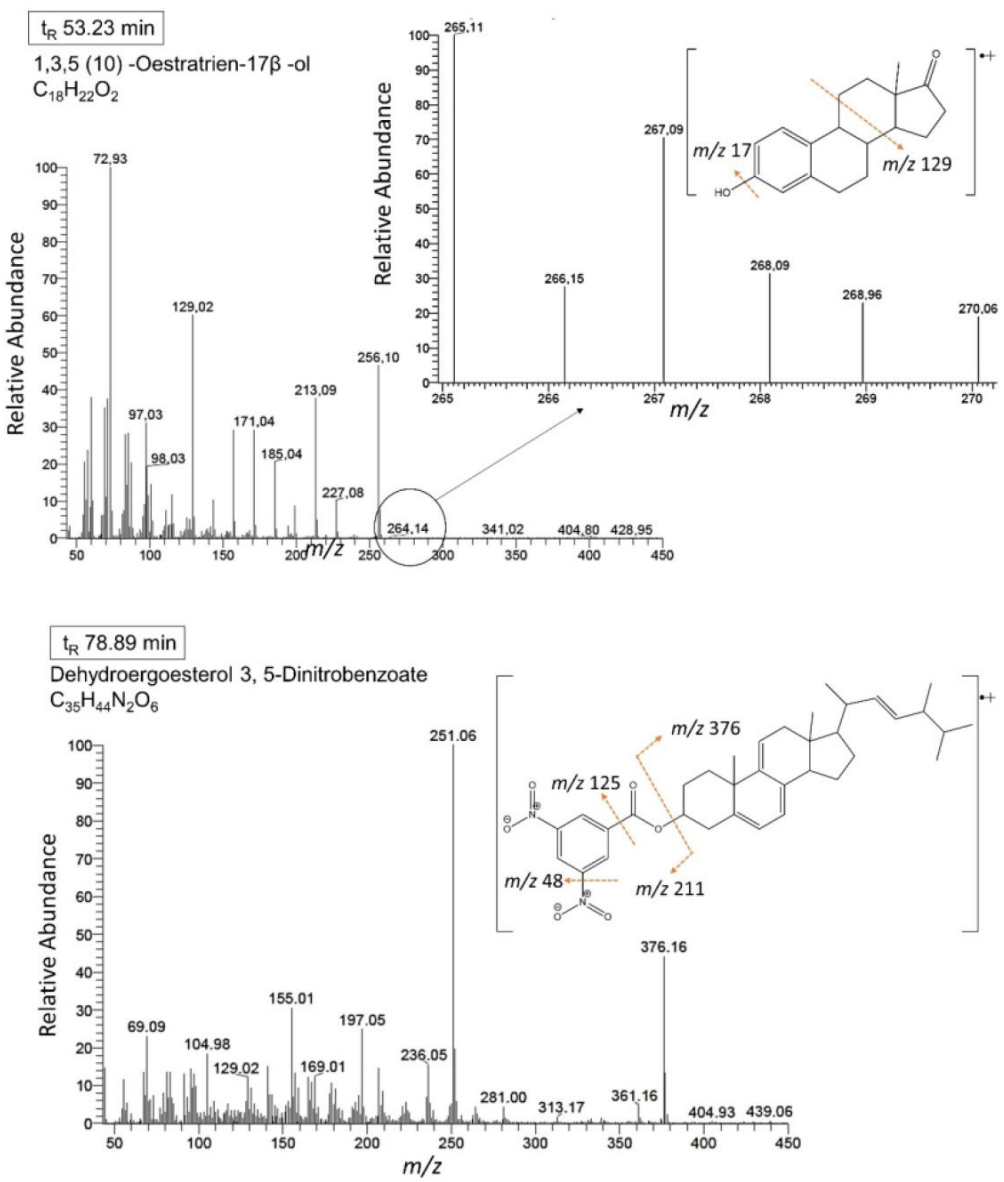

Figure 7. Mass spectra of the peaks with retention time of 53.23 and 78.89 min obtained after extraction from wheat subjected to bad storage conditions for 60 days. GC-MS in splitless mode with ionization energy of $70 \mathrm{eV}$ and scanning range between $\mathrm{m} / \mathrm{z} 43-450$.

extraction with ethyl acetate followed by GC-MS, without the need for microbiological analysis or very expansive technique as HPLC-MS/QTOF analysis, used in the present work as a comparative method.

\section{Supplementary Information}

Supplementary information is available free of charge at http://jbcs.sbq.org.br as PDF file. 
Table 2. Fusarium oxysporum metabolites identified in extracts of ethyl acetate of fungus cultivated in potato dextrose agar (PDA) and Czapek-Dox agar (CDA) media and in wheat samples

\begin{tabular}{|c|c|c|c|c|c|c|c|}
\hline Compound & Sum formula & $\begin{array}{l}\text { Retention } \\
\text { time / min }\end{array}$ & $\begin{array}{l}\text { Monoisotopic } \\
\text { mass }\end{array}$ & $\begin{array}{c}\mathrm{M}+\mathrm{H} \\
\text { (HPLC-MS/ } \\
\text { QTOF) }\end{array}$ & $\begin{array}{c}\mathrm{M}^{+} \bullet \\
(\mathrm{GC}-\mathrm{MS})\end{array}$ & $\begin{array}{l}\text { Percentage error } \\
\text { (HPLC-MS/ } \\
\text { QTOF) }\end{array}$ & $\begin{array}{c}\text { Percentage } \\
\text { error } \\
\text { (GC-MS) }\end{array}$ \\
\hline 13-Docosenamide & $\mathrm{C}_{22} \mathrm{H}_{43} \mathrm{NO}$ & 33.99 & 338.137 & 338.3407 & 337.86 & 0.06 & 0.08 \\
\hline Fusaric acid & $\mathrm{C}_{10} \mathrm{H}_{13} \mathrm{NO}_{2}$ & 34.96 & 179.095 & 180.1010 & 179.11 & 0.56 & $8.38 \times 10^{-3}$ \\
\hline $\begin{array}{l}\text { 2-Pyridinecarboxylic acid, } \\
\text { 5-(4-hydroxybutyl) }\end{array}$ & $\mathrm{C}_{10} \mathrm{H}_{13} \mathrm{NO}_{3}$ & 36.33 & 195.09 & 196.0966 & 195.17 & 0.52 & 0.04 \\
\hline $\begin{array}{l}\text { Cyclopentane } \\
\text { 1,1'-[3-(2-cyclopentylethylidene)- } \\
\text { 1,5-pentanediol] }\end{array}$ & $\mathrm{C}_{22} \mathrm{H}_{38}$ & 45.15 & 302.297363 & 303.6464 & 302.75 & 0.45 & 0.15 \\
\hline $\begin{array}{l}\text { Isobutyl 3-(perhydro-5-oxo-2-furyl) } \\
\text { propionate }\end{array}$ & $\mathrm{C}_{11} \mathrm{H}_{18} \mathrm{O}_{4}$ & 36.04 & 214.120514 & 215.0632 & 214.85 & 0.44 & 0.34 \\
\hline HT-2 toxin & $\mathrm{C}_{22} \mathrm{H}_{32} \mathrm{O}_{8}$ & 36.76 & 424.21 & 425.2132 & 424.82 & 0.24 & 0.14 \\
\hline $\begin{array}{l}\text { Spiro[4,5]dec-7-ene, 1,8-dimethyl- } \\
\text { 4-(1-methylethenyl) }\end{array}$ & $\mathrm{C}_{15} \mathrm{H}_{24}$ & 41.17 & 204.187805 & 205.1948 & 204.14 & 0.49 & 0.02 \\
\hline $1,3,5(10)$-Oestratrien-17 $\beta$-ol & $\mathrm{C}_{18} \mathrm{H}_{22} \mathrm{O}_{2}$ & 53.23 & 270.162 & 271.0201 & 270.06 & 0.32 & 0.04 \\
\hline$\underline{\text { Dehydroergosterol 3,5-dinitrobenzoate }}$ & $\mathrm{C}_{35} \mathrm{H}_{44} \mathrm{~N}_{2} \mathrm{O}_{6}$ & 78.89 & 588.32 & 589.7279 & a & 0.24 & a \\
\hline
\end{tabular}

${ }^{a}$ Not shown due to the high ionization energy used. HPLC-MS/QTOF: high-performance liquid chromatography mass spectrometry coupled to a quadrupole time-of-flight; GC-MS: gas chromatography mass spectrometry.

\section{Acknowledgments}

The authors are grateful to CAPES, CNPq, and Fundação Araucária for the financial support.

\section{References}

1. Brock, T. D.; Madigan, M. T.; Martinko, J. M.; Parker, J.; Biology of Microorganisms, $7^{\text {th }}$ ed.; Prentice-Hall International: Upper Saddle River, NJ, USA, 1994, p. 909.

2. Zain, M. E.; J. Saudi Chem. Soc. 2011, 15, 129.

3. Bebber, D. P.; Ramotowski, M. A. T.; Gurr, S. J.; Nat. Clim. Change 2013, 3, 985.

4. Marroquín-Cardona, A. G.; Johnson, N. M.; Phillips, T. D.; Hayes, A. W.; Food Chem. Toxicol. 2014, 69, 220.

5. Santin, E.; Maiorka, A.; Zanella, I.; Magon, L.; Cienc. Rural 2000, 31, 185.

6. Pereira, M. J. Z.; Ramalho, M. A. P.; Abreu, Â. F. B.; Cienc. Agrotecnol. 2011, 35, 940.

7. Nielsen, K. F.; Smedsgaard, J.; Larsen, T. O.; Lund, F.; Thrane, U.; Frisvad, J. C. In Fungal Biotechnology in Agricultural, Food and Environmental Application; Arora, D. K., ed.; Marcel Dekker: New York, 2004, p. 19-35.

8. Cirigliano, A. M.; Rodriguez, M. A.; Gagliano, M. L.; Bertinetti, B. V.; Godeas, A. M.; Cabrera, G. M.; J. Chromatogr. A 2016, 1439, 97.

9. Saldan, N. C.; Almeida, R. T. R.; Avíncola, A.; Porto, C.; Galuch, M. B.; Magon, T. F. S.; Pilau, E. J.; Svidzinski, T. I. E.; Oliveira, C. C.; Food Chem. 2018, 241, 113.

10. Schollenberger, M.; Lauber, U.; Jara, H. T.; Suchy, S.; Drochner, W.; Müller, H. M.; J. Chromatogr. A 1998, 815, 123.
11. Qian, M.; Zhang, H.; Wu, L.; Jin, N.; Wang, J.; Jiang, K.; Food Chem. 2015, 166, 23.

12. Engström, K.; Phytochemistry 1998, 47, 990.

13. Nielsen, K. F.; Smedsgaard, J.; J. Chromatogr. A 2003, 1002, 111.

14. Richard, J. L.; Thurston, J. R.; Diagnosis of Mycotoxicoses, $1^{\text {st }}$ ed.; Martinus Nijhoff Publishers: Madison, USA, 1986.

15. Uranga, C. C.; Beld, J.; Mrse, A.; Guerrero I. C.; Burkart, M. D.; Martínez, R. H.; Biochem. Biophys. Res. Commun. 2016, 472, 339.

16. Bankole, S. A.; Schollenberger, M.; Drochner, W.; J. Food Compos. Anal. 2010, 23, 837.

17. Espinel-Ingroff, A.; Kerkering, T. M.; J. Clin. Microbiol. 1991, 29, 393.

18. Foo, Y.; Chin, S.; Perlmutter, P.; Marriott, P. J.; J. Chromatogr. A 2015, 1387, 104.

19. Pereira, V. L.; Fernandes, J. O.; Cunha, S. C.; Food Chem. 2015, $182,143$.

20. Rodríguez-Carrasco, Y.; Moltó, J. C.; Berrada, H.; Mañes, J.; Food Chem. 2014, 146, 212.

21. Fumero, M. V.; Sulyok, M.; Chulze, S.; Food Addit. Contam., Part A 2016, 33, 147.

22. Fumero, M. V.; Reynoso, M. M.; Chulze, S.; Int. J. Food Microbiol. 2015, 199, 86.

23. Gourama, H.; Bullerman, L. B.; J. Food Prot. 1995, 58, 1395.

Submitted: March 6, 2018 Published onlline: July 27, 2018 